\title{
The Study on Consumer Behavior of Online Car-Hailing Platform and their Influencing Factors - Case Study of Didi Chuxing in China
}

\author{
Sihao $\mathrm{Gu}^{1, \mathrm{a}}$, Weilun Huang ${ }^{1, \mathrm{~b},{ }^{*}}$ \\ ${ }^{1}$ School of Finance, Wenzhou Business College, China \\ a760469305@qq.com, bhuangwl@wzbc.edu.cn \\ ${ }^{*}$ Corresponding author
}

Keywords: Online Car-Hailing Platform; Didi Chuxing; Consumer Behavior

\begin{abstract}
The purpose of this paper is to explore the behavior of consumer on the online car-hailing platform and its influencing factors based on online car-hailing types (Premier, Taxi, Express and Hitch) and analyze the differences brought by the age and monthly income of the consumer. The results from the questionnaire analysis are: 1 . The consumption frequency and amount of consumers (consumers behavior) on the online car-hailing platform are different with their consumption type. For example, the average monthly frequency and amount of Taxi (Express) consumers are 3.78 (5.58) and RMB 85.90 (RMB 73.13). 2. The significant influencing factors for consumer behavior are different with their consumption type. As the significant influencing factors of Premier consumers' behavior are price, quality, risk and community, but none for Taxi consumers' behavior. 3 . The age and monthly income of consumers would be significantly different with consumer behaviors of online car-hailing platform and their influencing factors, but none for their gender.
\end{abstract}

\section{Introduction}

The online car-hailing has been the main mode of transportation for people in many countries now, and its platform is the medium for its consumers' behavior. However, there are still few papers discussing consumer behavior of online car-hailing platform. Therefore, the purpose of this paper is to explore behavior of consumers on the online car-hailing platform and its influencing factors. Behavior of consumers on the online car-hailing platform is defined in this paper as a new trip mode for consumers with travel demands to make a reservation for the private car owner or taxi driver via the online car-hailing platform. According to iiMedia Research (2018), China's online car-hailing consumers have reached 435 million in 2017, an increase of 24.1\% compared with 365 million in 2016. Qianzhan Industry Research Institute (2018) has shown that Uber, Lyft, Grab, Ola, Uber, 99, Taxify, Careem, Didi International and other online car-hailing platforms serve more than $80 \%$ of population in the world, covering more than 1,000 cities. Jiang and Zhu (2018) explored the influencing factors on adoption intention of online car-hailing consumers including effort expectancy, social influence and perceived risk.

The behaviors of consumers on the online car-hailing platform should be different with their different service types, so this paper will explore the consumer behavior and its influencing factors according to various car-hailing types provided by the Chinese online car-hailing platform. For example, the car-hailing types provided by China's online car-hailing platform are divided into four categories: Premier, Taxi, Express and Hitch. The major differences among their car-hailing types are price and quality. China's four types of online car-hailing which ranked by the price (driving the same distance and from high to low) are: Premier, Taxi, Express and Hitch. Premier has the best service quality for its better car, smell-free, neat and tidy driver, and baggage pickup, and the quality of other three types are similar. The perceived risks on the types of online car-hailing are different, as there are several vicious crimes encountered by Hitch consumers in 2018, and Hitch has been stopped on August 27th, 2018. But Hitch was in operation when the questionnaire survey time of this paper, so Hitch consumers' behavior is still within the scope of this paper. 
This paper thought the factors influencing consumer's behavior of the online car-hailing platform should be its price, quality, perceived risk and community. The focuses for its price are the discount and peak pricing policy which were set by the online car-hailing platform. For example, Didi Chuxing which is the online car-hailing platform in China allowed and encouraged payment via WeChat in 2014 so that consumers can get a random discount of RMB 12-20 each time. Its peak pricing policy refers to the policy of higher price during peak hours and peak sections. Jiang and Zhu (2018) and Cheng, et al. (2017) found the impact of online car-hailing fare per mileage on consumer's switch intention of trip mode and consumer's willingness of the online car-hailing platform. Its quality depends on the handling time, vehicle quality, driver's service quality and platform usability. According to the regulation set by Didi Chuxing, if the driver could not arrive within 10 minutes after the scheduled time, the consumer could cancel this order for free. Besides, vehicle of Express is divided into two types: ordinary and superior. Premier has three types: comfortable, business and administrative. Cheng, et al. (2017) found that service quality of drivers has a significant impact on user's switch intention of trip mode. Both Jacquet (2018) and Weng, et al. (2017) assume its platform usability has a significant influence on behavior of consumers on the online car-hailing platform.

The focuses of its perceived risk are the operational risk, privacy risk and crime risk of the online car-hailing platform. Its operational risk refers to the risk of property loss caused by improper operation of consumers on the online car-hailing platform. Its privacy risk refers to the risk of privacy leakage caused by consumers submitting their personal information like destination and telephone number to the platform or the driver. Its crime risk refers to the risk to suffer the criminal codes (as theft, robbery and etc.) during the process of enjoying online car-hailing. Redmiles (2013) assumes that perceived risk is an important factor affecting behavior of consumers on the online car-hailing platform. Jiang and Zhu (2018) found its perceived risk has a negative impact on user's consumption willingness. The focuses of its community impact are the preferences for online car-hailing by the community around consumers and the media promotion. For example, on March 8th, 2018, Didi Chuxing released the first "Employment Report of Women on DiDi Platform" to emphasize the company's social responsibility. Jiang and Zhu (2018) thought its community influence would positively affect consumption willingness of consumers on the online car-hailing platform.

Another focus of this paper is to explore the impacts of the gender, age and monthly income of consumer on the consumer's behavior of the online car-hailing platform and its influencing factors. For example, the 36kr Group (2018) found the proportion of women who choose online car-hailing as the primary trip mode is greater than that of men. According to the Data from the Qianzhan Industry Research Institute (2018), the proportion of online car-hailing consumers whose age is 25-35 is the highest (50.9\%). The higher monthly income of consumer is, the higher frequency of online car-hailing would be. For further study, this paper takes Didi Chuxing in China as an example for it is the online car-hailing platform with the highest market share in China, CNIT-Research (2016) found that the market share of Didi Chuxing was 94.6\% in 2016. The paper is divided into four parts: Introduction, literature review, the analysis of the questionnaire results and the conclusion.

\section{Literature review}

According to literature review, it is known that behavior of consumers on the online car-hailing platform should be an important research topic but there are still few papers discussing behavior of consumers on the online car-hailing platform. This paper surveyed the existing literature in China and found that the paper titled "online car-hailing" dates back to 2015 and there have been 1,427 papers up to September 29th, 2018. These study focus on the status and questions of online car-hailing, taxi industry and sharing economy. There are 35 papers titled "the consumer of online car-hailing" with the focus on the perceived risk, laws and policies of online car-hailing. At present, there are still few papers studying behavior of consumers on the online car-hailing platform and its influencing factors. Therefore, the focus of this paper is to explore behavior of consumers on the online car-hailing platform and its influencing factors so as to supplement the inadequacies of the literature as well as providing suggestions for individuals, enterprises and government-related policy. 
The variables about behavior of consumers on the online car-hailing platform are defined as consumer's monthly consumption frequency and amount on the four types of online car-hailing provided by the Didi Chuxing platform. For example, Zhao (2017) defines consumer continuous use behavior as the used time, the frequency every three months and the amount of the online car-hailing platform. Sakunlertvattana (2016) divides consumer's consumption frequency of the online car-hailing platform into once in 1, 3, 5 days.

The variables about price of the online car-hailing platform are defined as the discount and peak pricing policy set by the Didi Chuxing. For example, Rao (2017) assumes that the price of the online car-hailing should include the discount, the fare per kilometer and in night. Jacquet (2018) found that peak price of UBER is much higher than that of Lyft.

The variables about quality of online car-hailing are defined as their handling time, vehicle quality, driver's service quality and usability of the online car-hailing platform. For example, Ross (2015) thought the service quality of the online car-hailing should include vehicle quality, driver's attitude and handling time. Rao (2017) believes that vehicle quality should include the model and decoration inside the car. Hussei (2015) thinks that the service quality of the online car-hailing driver should include the driver's appearance and carry-on first aid kit. Rao (2017) believes that usability of the online car-hailing platform should include the conveniences of conserving online car-hailing and making complaints.

The variables about the perceived risk of the online car-hailing platform are defined as the criminal codes suffered by consumers while they are using Didi Chuxing, property loss due to customers' improper use of the Didi Chuxing platform and personal privacy leakage from the driver or Didi Chuxing platform. For example, Li (2018) believes that the perceived risk of the online car-hailing platform should include the risks of late, crime, payment and privacy.

The variables about the community impact of the online car-hailing platform are defined as neighboring groups using Didi Chuxing and recommending it as well as media promotion. Sakunlertvattana (2016) defines its community as family, friends, advertisement and social network. Lan (2016) defines community impact as recommendation from close relatives and friends using the online car-hailing platform as well as the acceptance of online car-hailing platform from the surrounding friends.

The variables about the individual variables are defined as consumer's gender, age and monthly income. For example, Cheng et al. (2017) studied the impact of discount on user's switch intention of trip mode with consumer's age, gender and monthly income as the variables. Zhang (2017) defines the consumer's individual variables as gender, age, annual income and vehicles.

\section{The Analysis of Questionnaire Result}

In June of 2018, the online questionnaire was conducted in China. The number of copies collected and completely filled out is 315. The descriptive statistic results of the respondents' individual variables are: 1 . Gender: women accounting for $49.21 \%$ and men accounting for $50.79 \%$. 2 . Age: The average age of respondents is 34.37 (standard deviation was 11.25). 3. Monthly income: The average monthly income of respondents is RMB 3,463.49 (standard deviation is 11.25)

The descriptive statistic results on the behavior variables of online car-hailing consumers are: 1 . Consumption frequency: The average consumption frequency of respondents using Premier, Taxi, Express and Hitch per month is 5.56 (standard deviation is 3.30), 3.78 (standard deviation is 3.34), 6.25 (standard deviation is 3.24) and 5.58 (standard deviation is 2.95). 2. Consumption amount: The average consumption amount of respondents using Express, Hitch, Premier and Taxi per month is RMB 71.11 (standard deviation is 47.97), RMB 48.76 (standard deviation is 48.62), RMB 73.13 (standard deviation is 43.68) and RMB 85.90 (standard deviation is 49.33).

The descriptive statistic results of the influencing factors of behavior of consumers on online car-hailing platform are: 1 . Most respondents approve its service quality, while least for the community impact. 2. As for online car-hailing price, most respondents agree that they will choose Didi Chuxing for its discount. 3. In terms of service quality, most respondents agree with the usability 
of online car-hailing platform. 4. As for its perceived risk, most respondents agree with the crime risk. 5. As for its community impact, most respondents agree with its media promotion.

The variance analysis (ANOVA) of behavior of consumers on online car-hailing platform and its influencing factors is sorted out in Table 1. From Table 1, the influencing factors of the four types of online car-hailing are different. For example, Premier has four significant influencing factors. Only consumption amount of Premier users is not affected by the service quality; Taxis are not significantly affected by the four influencing factors with exception of consumption frequency of Taxi users that is significantly affected by community impact; Consumption amount of Express consumers is significantly influenced by price and community impact while consumption frequency of Express users is only influenced by price; Hitch is remarkably influenced by the factors while its fare is not affected by community impact. Price is positively correlated with consumption amount Hitch users. Community impact is the most influential factor to customer behavior excepting for consumption amount of Taxi and Hitch users.

Table 1 Variance Analysis of Its Influencing Factors on the Behavior of Online car-hailing Consumers

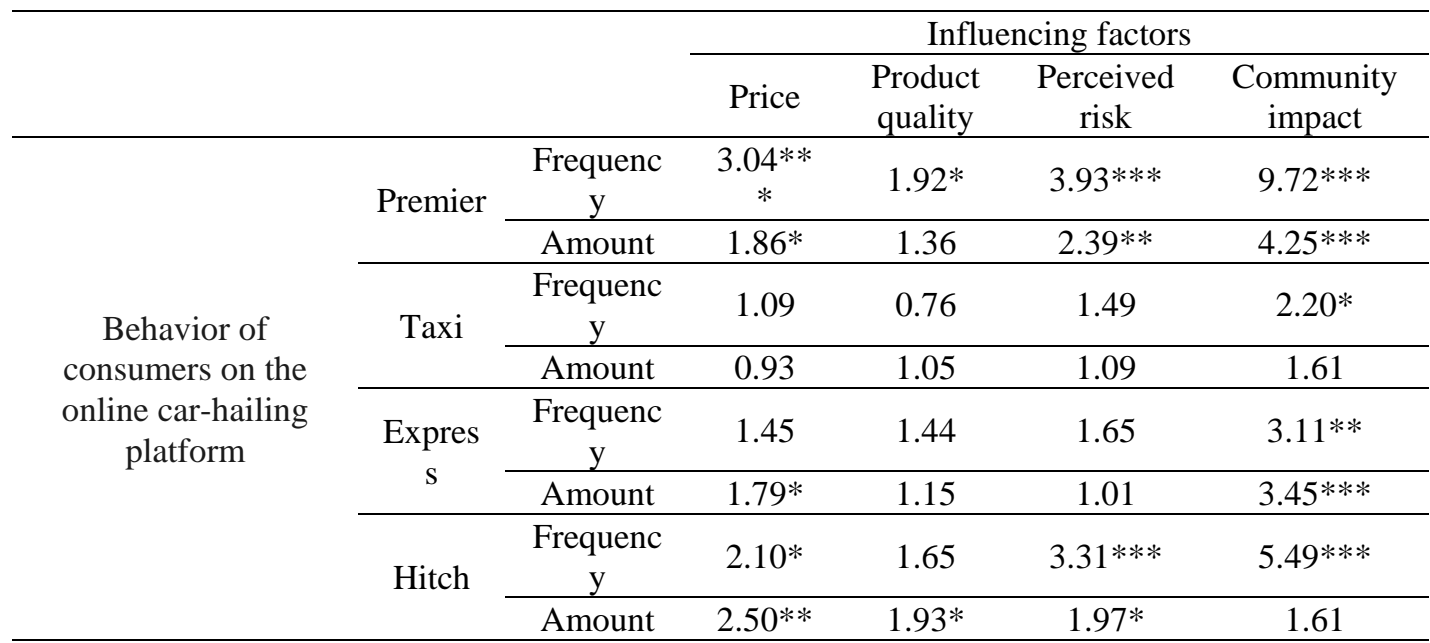

To further understand the questionnaire results, the impact of consumer's gender, age and monthly income on behavior of consumers on online car-hailing platform and its influencing factors are explored in this paper. According to the survey statistics, consumer's gender has no significant impact on behavior of consumers on online car-hailing platform and its influencing factors. The variance analysis of individual variables (age and monthly income) on the behavior of online car-hailing consumers and its influencing factors is shown in Table 2. From Table 2, consumer's age and monthly income have significant influence on the consumer's behavior of online car-hailing platform. For example, the age and monthly income of consumer influence Premier customer behavior significantly and are all positively correlated. Besides, the age has significant influence on consumption frequency of Express consumers and is positively correlated. The age and monthly income have significant influences on consumption frequency of Hitch customers and are also positively correlated. However, the impact of individual variables on Taxi consumer behavior is not significant as well as the impact on the four influencing factors. Only monthly income has a significant impact on perceived risk. 
Table 2 Variance Analysis of Individual Variables (Age and Monthly Income) on the Behavior of Online Car-hailing Consumers and its Influencing Factors

\begin{tabular}{|c|c|c|c|c|}
\hline & & & \multicolumn{2}{|c|}{ Individual variables } \\
\hline & & & Age & Monthly income \\
\hline \multirow{8}{*}{$\begin{array}{l}\text { Behavior of consumers on the } \\
\text { online car-hailing platform }\end{array}$} & \multirow{2}{*}{ Premier } & Frequency & $9.09 * * *$ & $4.44 *$ \\
\hline & & Amount & $3.87 * *$ & $4.33 *$ \\
\hline & \multirow{2}{*}{ Taxi } & Frequency & 2.30 & 1.80 \\
\hline & & Amount & 0.71 & 2.30 \\
\hline & \multirow{2}{*}{ Express } & Frequency & $5.02 * *$ & 2.08 \\
\hline & & Amount & 0.89 & 1.13 \\
\hline & \multirow{2}{*}{ Hitch } & Frequency & $6.71 * * *$ & $5.01 * *$ \\
\hline & & Amount & 1.11 & 2.33 \\
\hline \multirow{4}{*}{ Influencing factors } & \multicolumn{2}{|c|}{ Price } & 0.78 & 1.03 \\
\hline & \multicolumn{2}{|c|}{ Service quality } & 0.96 & 1.01 \\
\hline & \multicolumn{2}{|c|}{ Perceived risk } & 1.51 & $4.33 *$ \\
\hline & \multicolumn{2}{|c|}{ Community impact } & 1.97 & 0.05 \\
\hline
\end{tabular}

Based on the above findings, the suggestions given by this paper are: 1 . It can be known from Table 1 and Table 2 that the significant influencing factors of the four types of services provided by the platform are different. Therefore, it is suggested in this paper that market segmentation should be conducted by the online car-hailing platform according to its differences of four services. For example, Premier consumers should focus on consumers who are older with higher monthly income and pay more attention to service quality.

2. From Table 1 and Table 2, the significant influencing factors on the behavior of Premier consumers would be are price, service quality, perceived risk, community impact and individual variables (age and monthly income). The reason is considered in this paper as the highest fare per mileage of the Premier. Therefore, Premier consumers would have the highest demands for related services, as its discount, driver service, vehicle quality, crime probability and media promotion. Therefore, it is suggested in this paper that dedicated service for consumers should be set by the online car-hailing platform. More high-end services, such as organizing car drivers to participate in professional training, regular vehicle cleaning and disinfection, exclusive car insurance and playing advertisements via media should be provided.

3. From Table 1 and Table 2, there is no significant influencing factor of Taxi consumers. The reasons behind it, believed in this paper, are that the online car-hailing platform is not the only access to Taxi and consumers do not have specific requirements for taxi. Therefore, it is suggested in this paper that there is no need for the online car-hailing platform to specifically promote the taxi service or re-evaluate the necessity of Taxi service.

4. From Tables 1 and 2, it is known that the significant influencing factors of Express consumer behavior are price and community impact. Therefore, it is suggested in the paper that coupons should be issued to consumers via the online car-hailing platform to encourage consumers to share good traveling experiences with relatives and friends. In addition, excellent drivers should be organized to hold advertising campaigns for vehicles with characteristics, making use of the news media to promote the social value of the online car-hailing platform.

5. From Table 1 and Table 2, the significant influencing factors of the behavior of Hitch consumers are price, perceived risk and community impact. However, due to several vicious crimes occurred in 2018, consumers have serious doubts about the safety of Hitch. Therefore, Hitch service has been canceled and benefits or reputation of the online car-hailing platform have been damaged. If PIC of the online car-hailing platform wants to re-launch Hitch service, it is suggested in the paper that the access conditions of Hitch should be improved by the online car-hailing platform (drivers with criminal tendency shall be banned from dipping a toe), the function of tracking the position of Hitch in real time should be launched as well as the one-button alarm function. 


\section{Summary}

With increasing popularity of the Internet and promotion of the online car-hailing platform, the public can provide online car-hailing (such as China's Premier, Express and Hitch) in a personal capacity at a lower cost, so that the online car-hailing supply increases; Besides, the online car-hailing platform reduces the search cost and transaction cost of consumers, which has increased consumers' demand for online car-hailing. Thus, the equilibrium number of online car-hailing markets has been on the rise and the equilibrium price has decreased, which in turn has caused the scale of the online car-hailing industry to expand continuously. Since the online car-hailing has been divided into four categories by the platform in China (Premier, Taxi, Express and Hitch), the price, service quality, perceived risk and community impact of these four different services are different. However, there are still few papers discussing the four categories. Therefore, the purpose of this paper is to explore behavior of customers on the online car-hailing platform and its influencing factors based on online car-hailing types (Premier, Taxi, Express and Hitch) and analyze difference caused by the age and monthly income.

According to literature review and statistical results of the questionnaire, it is found that: 1 . Behavior of consumers on the online car-hailing platform should include consumption frequency and consumption amount of Premier, Taxi, Express and Hitch users. 2. The significant factors affecting the behavior of Premier consumers are price, service quality, perceived risk and community impact. 3 . There is no significant factor affecting behavior of Taxi consumers. 4. Significant factors influencing the behavior of express consumers are price and community impact. 5. Significant factors influencing behavior of Hitch consumers are price, service quality, perceived risk and community impact. 6. Age should have a significant impact on the behavior of Premier, Express and Hitch consumers; Monthly income should have a significant impact on the behavior of Premier and Hitch consumers, while gender has no significant impact on behavior of consumers on online car-hailing platform.

Further study directions of this paper include considering customer satisfaction in the influencing factors and covering areas in the service quality. For example, Hussein (2016) views that customer satisfaction is an indicator for predicting purchases of future consumers. Jacquet (2016) believes that covering areas can enable consumers to enjoy services in more places.

\section{References}

[1] Media Research (2018). 2017-2018 special report on the research of China's online car-hailing industry. China. Guangdong.

[2] Jiang, y. and Zhu, W. (2018). Study on Users' Adoption Behavior for Internet Chauffeured Car. International Journal of e-Education, e-Business, e-Management and e-Learning. 8(2):82-90, June 2018.

[3] Cheng, X. et al. (2017). Does Subsity Work? An Investigation of Post-Adoption Switching on Car-Hailing Apps. Journal of Electronic Commerce Research, VOL 18(4): 317-329, 2017,

[4] Jacquet, A. (2018),A Power Struggle in the Taxi Industry: A Challenge for Uber and Lyft Regarding Their Service Clauses. PM World Journal Vol. VII, Issue II - February 2018.

[5] Redmiles, E. (2013). Human Factors and Requirements for A Successful Mobile Platform Dynamic Taxi-share System in the USA. University of Maryland Honours Thesis. Retrieved February 5, 2014.

[6] 36kr Group（2018） , 2018 online car-hailing user research report. 36kr Group : China, Beijing. 